\title{
Fecal Occult Blood Test and Gastrointestinal Parasitic Infection
}

\author{
Majed H. Wakid \\ Department of Medical Laboratory Technology, Faculty of Applied Medical Sciences, King AbdulAziz University-Jeddah, \\ P.O. Box 80324, Jeddah 21589, Saudi Arabia \\ Correspondence should be addressed to Majed H. Wakid, mwakid@kau.edu.sa
}

Received 27 May 2010; Accepted 23 June 2010

Academic Editor: Wej Choochote

Copyright ( 92010 Majed H. Wakid. This is an open access article distributed under the Creative Commons Attribution License, which permits unrestricted use, distribution, and reproduction in any medium, provided the original work is properly cited.

Stool specimens of 1238 workers in western region of Saudi Arabia were examined for infection with intestinal parasites and for fecal occult blood (FOB) to investigate the possibility that enteroparasites correlate to occult intestinal bleeding. Direct smears and formal ether techniques were used for detection of diagnostic stages of intestinal parasites. A commercially available guaiac test was used to detect fecal occult blood. $47.01 \%$ of the workers were infected with intestinal parasites including eight helminthes species and eight protozoan species. The results provided no significant evidence $(P$-value $=0.143)$ that intestinal parasitic infection is in association with positive guaiac FOB test.

\section{Introduction}

Human infections due to intestinal parasites caused by helminths and protozoa are among the most prevalent infections in developing and tropical countries causing a significant morbidity and mortality [1]. Human intestinal helminths include the nematodes (roundworms), the trematodes (flukes), and the cestodes (tapeworms). The human intestinal protozoa include nonpathogenic and pathogenic amoebae, nonpathogenic and pathogenic flagellates, and a pathogenic ciliate, in addition to human intestinal coccidian parasites.

Fecal occult blood (FOB) refers to a nonvisible blood in the stool. Although the FOB test was developed to specifically screen for colon cancer $[2,3]$, there are various causes of positive FOB including infection with some intestinal parasites $[4,5]$. From parasitologic point view, most of the published studies concerned with determination of FOB in patients infected with Trichuris trichiura, Hook worm, Schistosoma spp. and Entamoeba histolytica [6-14]. The aim of the present study was to determine if there is correlation between positive FOB and any of the common intestinal parasitic infection in Saudi Arabia.

\section{Materials and Methods}

2.1. Samples Collection. Stool samples were obtained randomly from 1238 workers in the western region of Saudi Arabia. Each worker was provided with a clean stool container and instructions for collection.

2.2. Parasitologic Examination. The direct smears and formal ether concentration techniques were used for detection of diagnostic stages of enteric parasites $[15,16]$.

Direct stool smears were performed by emulsifying $2 \mathrm{mg}$ of stool uniformly in a drop of saline or iodine on a microscope slide, then covered with cover glasses and scanned microscopically.

Formal ether concentration technique was performed by emulsifying $2 \mathrm{~g}$ of stool in $15 \mathrm{~mL}$ of $10 \%(\mathrm{v} / \mathrm{v})$ formal-saline. In unpreserved specimens, the suspension was allowed to stand for $30 \mathrm{~min}$, then strained through two layers of gauze into a $15 \mathrm{~mL}$ conical centrifuge tube and centrifuged at $2000 \mathrm{rpm}$ for $5 \mathrm{~min}$. When needed, washing step was repeated until supernatant becomes clear. The sediment was resuspended with $10 \mathrm{~mL}$ of $10 \%(\mathrm{v} / \mathrm{v})$ formal-saline, then $3 \mathrm{~mL}$ of diethyl ether was added. The tube was shaken vigorously for $30 \mathrm{sec}$ and centrifuged at $2000 \mathrm{rpm}$ for $5 \mathrm{~min}$. 
TABLE 1: FOB finding in negative (control) and positive parasitic infections.

\begin{tabular}{|c|c|c|c|}
\hline & & & \\
\hline & Negative $(n=656)$ & Positive $(n=582)$ & \\
\hline +Ve FOB & $170(25.91 \%)$ & $130(22.43 \%)$ & $P$-value $=0.0143$ \\
\hline - Ve FOB & $486(74.09 \%)$ & $452(77.57 \%)$ & \\
\hline
\end{tabular}

TABLE 2: FOB results in correlation with infection of detected parasites. $n=$ number of infected cases. ${ }^{*}$ All samples were negative for FOB test.

\begin{tabular}{|c|c|c|c|c|c|}
\hline \multirow{2}{*}{ Parasite $(n)$} & \multicolumn{2}{|c|}{ (Single infection) } & \multicolumn{2}{|c|}{ (Mixed infection) } & \multirow{2}{*}{$P$-value } \\
\hline & $+\mathrm{Ve}$ FOB & - Ve FOB & $+\mathrm{Ve}$ FOB & - Ve FOB & \\
\hline Hookworm (184) & 32 & 67 & 35 & 50 & 0.213 \\
\hline T. trichiura (123) & 14 & 37 & 19 & 53 & 0.896 \\
\hline A. lumbricoides (11) & 2 & 4 & 1 & 4 & 0.621 \\
\hline H. nana (10) & 0 & 4 & 0 & 6 & $*$ \\
\hline S. mansoni (14) & 2 & 1 & 4 & 7 & 0.347 \\
\hline S. stercoralis (20) & 1 & 4 & 3 & 12 & 1.0 \\
\hline E. vermicularis (2) & 0 & 0 & 0 & 2 & $*$ \\
\hline T. saginata (2) & 0 & 1 & 0 & 1 & $*$ \\
\hline E. histolytica (43) & 1 & 0 & 14 & 28 & 0.167 \\
\hline G. lamblia (39) & 4 & 17 & 3 & 15 & 0.847 \\
\hline B. hominis (245) & 16 & 109 & 17 & 103 & 0.754 \\
\hline Nonpathogenic protozoa (156) & 12 & 33 & 19 & 92 & 0.176 \\
\hline
\end{tabular}

The fecal debris layer was loosened by wooden applicator stick and the tube rapidly inverted to discard the top three layers while the sediment remained at the bottom. A drop of iodine was mixed with the sediment, then transferred to a microscope slide, covered with a cover glass, and scanned microscopically.

2.3. Occult Blood Test. Each stool sample was processed to detect occult blood using a guaiac-based test (Hema-Screen, Stanbio, Texas, USA) by spreading thin smear of stool according to the manufacturer instructions. Hema-Screen can detect $10 \mathrm{mg}$ of hemoglobin per gram of feces. A positive reaction is indicated by the appearance of a blue-green color between 30 seconds and two minutes after addition of two drops of developer reagent. The occult blood tests were achieved by a technologist blinded to parasitologic results.

2.4. Statistical Analysis. Data analysis was performed using SPSS V17.0 statistic Package. The Chi square test was used for determining the significance of association between intestinal parasitic infection and FOB test finding. A $P$-value level of significance was 0.05 .

The study was approved by the Research and Ethics Committee of the Faculty of Applied Medical Sciences, King Abdulaziz University, Jeddah, Saudi Arabia.

\section{Results}

The enrolled 1238 workers in this study were males within the age of $17-70$. Six hundred and fifty six (52.99\%) cases without intestinal parasitic infection were the control of the study.

Five hundred and eighty two $(47.01 \%)$ were infected with intestinal parasites. These parasites included hookworms, Trichuris trichiura, Ascaris lumbricoides, Hymenolepis nana, Schistosoma mansoni, Strongyloides stercoralis, Enterobius vermicularis, Taenia saginata, E. histolytica, Giardia lamblia, Blastocystis hominis, and several nonpathogenic intestinal protozoan parasites (Entamoeba coli, Endolimax nana, Entamoeba hartmanii, Iodamoeba butschlii, and Chilomastix mesnili).

Of 582 infected cases, the positive rate for occult blood was $130(22.43 \%)$. There were $170(25.91 \%)$ positive fecal blood results in the 656 control group (Table 1).

There was no significant association between positive FOB test and infection with intestinal parasites $(P=0.0143)$. Results of FOB for each detected parasitic infection alone or in presence of other parasites as mixed infection are illustrated in Table 2.

\section{Discussion}

Detecting fecal occult blood by a guaiac-based test is still the most widely used test in medical laboratories in Jeddah. This test depends on the peroxidase-like activity of hemoglobin in catalyzing the oxidation by peroxide of a chromogen. Dietary and medication restrictions are recommended with this test to decrease false positive and false negative results [5]. Immunochemical FOB test, which does not require dietary restrictions, is more expensive and not used widely in Jeddah. An earlier study showed that both tests had a similar positive 
predictive value [17]. According to that, guaiac-based test was suitable for our design during this study to resemble the situations during routine analysis in medical laboratories in Jeddah, regardless of dietary and medication restrictions, which is not followed by the majority of ordinary population in Jeddah and regardless of infection intensity, which is not performed with routine FOB testing.

During this study several pathogenic and nonpathogenic enteroparasites were detected and investigated for correlation to FOB positivity. There was no significant difference in FOB positivity between infected and noninfected samples (Tables 1 and 2).

The common parasites which were detected and can cause dysentery or blood loss were Trichuris trichiura, hookworms, Schistosoma mansoni, and E. histolytica. Previous studies illustrated that occult blood is not correlated with T. trichiura infection, unless there is a Trichuris dysentery syndrome (TDS) $[6,7,10]$. Similarly for hookworms, they do not lead to a significant occult gastrointestinal bleeding unless high-burden infection occurs [11-13]. Same observation was reported concerning Schistosoma spp. that infects humans and causes anemia $[9,14,18-20]$. A previous study reported four asymptomatic cases with positive FOB test and amebic colitis due to E. histolytica [8].

In the present study, all samples with E. vermicularis and T. saginata reacted negatively with FOB test. In a previous study, E. vermicularis (pinworm) gave positive FOB but in a case with colon carcinoma [21].

The importance of the recognition of nonpathogenic (commensals) amoebae and flagellates lies in the fact that these organisms are indicative of fecal-oral transmission having occurred. When these organisms are found in stool samples, it is important to be on the lookout for the possible presence of pathogenic species. B. hominis represented the highest infection rate (19.79\%); however, describing this parasite as pathogenic is still controversial [22-24].

In summary, the present study demonstrated that positive guaiac-based FOB test during routine analysis in Jeddah could not be correlated with the intestinal parasitic infection. However, as guaiac-based FOB test is widely used in Jeddah, more awareness for the public about importance of restricting some foods and drugs intake prior FOB test is needed.

\section{Acknowledgments}

This study was funded by King Abdulaziz University, Jeddah Saudi Arabia. Thanks are extended to King Fahad Medical Research Centre and Faculty of Applied Medical Sciences for providing the place for practical work of this study. The author would like to thank Dr. Ezz AbdulFatah from statistics department for his assistance in the statistical analysis of this study.

\section{References}

[1] WHO, http://www.who.int/topics/intestinal_diseases_parasitic len.

[2] D. Wu, D. Erwin, and G. L. Rosner, "A projection of benefits due to fecal occult blood test for colorectal cancer," Cancer Epidemiology, vol. 33, no. 3-4, pp. 212-215, 2009.
[3] F. Jenkinson and R. J. C. Steele, "Colorectal cancer screeningmethodology," Surgeon, vol. 8, no. 3, pp. 164-171, 2010.

[4] S. H. Mitchell, D. C. Schaefer, and S. Dubagunta, "A new view of occult and obscure gastrointestinal bleeding," American Family Physician, vol. 69, no. 4, pp. 875-881, 2004.

[5] M. Beg, M. Singh, M. K. Saraswat, and B. B. Rewari, "Occult gastrointestinal bleeding: detection, interpretation and evaluation," Journal Indian Academy of Clinical Medicine, vol. 3, no. 2, pp. 153-158, 2002.

[6] M. S. Khuroo, M. S. Khuroo, and N. S. Khuroo, "Trichuris dysentery syndrome: a common cause of chronic iron deficiency anemia in adults in an endemic area (with videos)," Gastrointestinal Endoscopy, vol. 71, no. 1, pp. 200-204, 2010.

[7] D. Wanachiwanawin, S. Wongkamchai, S. Loymek et al., "Determination of fecal occult blood in primary schoolchildren infected with Trichuris trichiura," Southeast Asian Journal of Tropical Medicine and Public Health, vol. 36, no. 5, pp. 11101113, 2005.

[8] M. Okamoto, T. Kawabe, K. Ohata et al., "Short report: amebic colitis in asymptomatic subjects with positive fecal occult blood test results: clinical features different from symptomatic cases," American Journal of Tropical Medicine and Hygiene, vol. 73, no. 5, pp. 934-935, 2005.

[9] J. F. Friedman, H. K. Kanzaria, L. P. Acosta et al., "Relationship between Schistosoma japonicum and nutritional status among children and young adults in Leyte, the Philippines," American Journal of Tropical Medicine and Hygiene, vol. 72, no. 5, pp. 527-533, 2005.

[10] S. M. Raj, "Fecal occult blood testing of Trichuris-infected primary school children in Northeastern Peninsular Malaysia," American Journal of Tropical Medicine and Hygiene, vol. 60, no. 1, pp. 165-166, 1999.

[11] R. J. Stoltzfus, M. Albonico, H. M. Chwaya et al., "Hemoquant determination of hookworm-related blood loss and its role in iron deficiency in African children," American Journal of Tropical Medicine and Hygiene, vol. 55, no. 4, pp. 399-404, 1996.

[12] A. Allemann, P. Bauerfeind, and N. Gyr, "Prevalence of hookworm infection, anaemia and faecal blood loss among the Yupno people of Papua New Guinea," Papua and New Guinea Medical Journal, vol. 37, no. 1, pp. 15-22, 1994.

[13] P. Bauerfeind, A. Allemann, and N. Gyr, "Differential diagnostic value of occult blood in stool in hookworm infection in the Third World. Study from Papua New Guinea," Zeitschrift fur Gastroenterologie, vol. 30, no. 11, pp. 791-794, 1992.

[14] F. A. Proietti and C. M. F. Antunes, "Sensitivity, specificity and positive predictive value of selected clinical signs and symptoms associated with Schistosomiasis mansoni," International Journal of Epidemiology, vol. 18, no. 3, pp. 680-683, 1989.

[15] M. H. Wakid, E. I. Azhar, and T. A. Zafar, "Intestinal parasitic Infection among food handlers in the Holy City of Makkah during Hajj season 1428 Hegira (2007G)," Journal of King Abdulaziz University Medical Sciences, vol. 16, no. 1, pp. 3952, 2009.

[16] M. H. Wakid, "Distribution of intestinal parasites among food handlers in Jeddah, Saudi Arabia," Journal of Parasitic Diseases, vol. 30, no. 2, pp. 146-152, 2006.

[17] C. W. Ko, J. A. Dominitz, and T. D. Nguyen, "Fecal occult blood testing in a general medical clinic: comparison between guaiac-based and immunochemical-based tests," American Journal of Medicine, vol. 115, no. 2, pp. 111-114, 2003.

[18] J. F. Friedman, H. K. Kanzaria, and S. T. McGarvey, "Human schistosomiasis and anemia: the relationship and potential 
mechanisms," Trends in Parasitology, vol. 21, no. 8, pp. 386392, 2005.

[19] H. K. Kanzaria, L. P. Acosta, G. C. Langdon et al., "Schistosoma japonicum and occult blood loss in endemic villages in Leyte, The Philippines," American Journal of Tropical Medicine and Hygiene, vol. 72, no. 2, pp. 115-118, 2005.

[20] T. Leenstra, L. P. Acosta, G. C. Langdon et al., "Schistosomiasis japonica, anemia, and iron status in children, adolescents, and young adults in Leyte, Philippines," American Journal of Clinical Nutrition, vol. 83, no. 2, pp. 371-379, 2006.

[21] S.-C. Lee, K.-P. Hwang, W.-S. Tsai, C.-Y. Lin, and N. Lee, "Detection of Enterobius vermicularis eggs in the submucosa of the transverse colon of a man presenting with colon carcinoma," American Journal of Tropical Medicine and Hygiene, vol. 67, no. 5, pp. 546-548, 2002.

[22] M. J. Zuckerman, H. Ho, L. Hooper, B. Anderson, and S. M. Polly, "Frequency of recovery of Blastocystis hominis in clinical practice," Journal of Clinical Gastroenterology, vol. 12, no. 5, pp. 525-532, 1990.

[23] H.-Y. Kuo, D.-H. Chiang, C.-C. Wang et al., "Clinical significance of Blastocystis hominis: experience from a medical center in northern Taiwan," Journal of Microbiology, Immunology and Infection, vol. 41, no. 3, pp. 222-226, 2008.

[24] Y. Sukthana, "Is Blastocystis hominis a human pathogenic protozoan?" The Journal of Tropical Medicine and Parasitology, vol. 24, no. 1, pp. 16-22, 2001. 

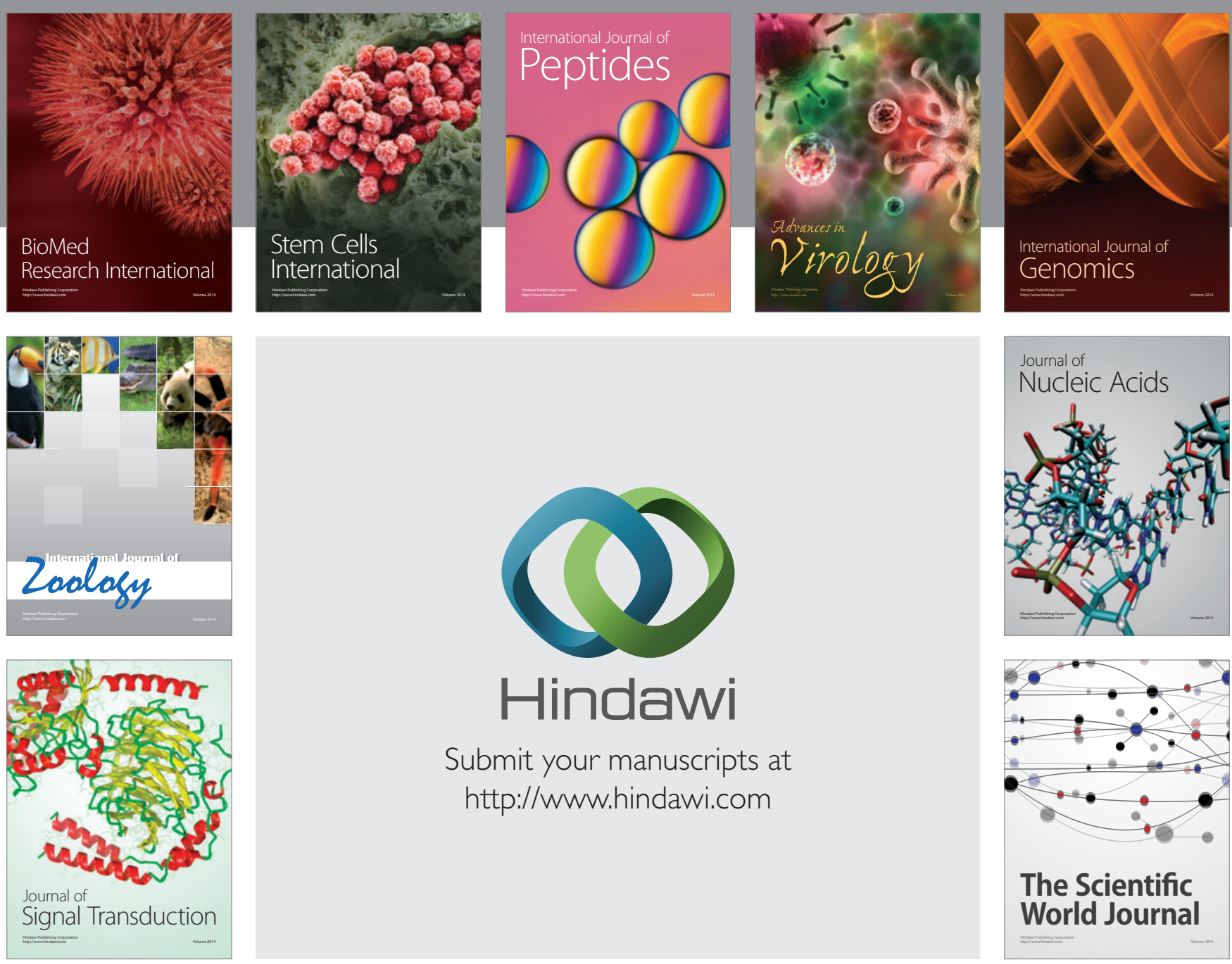

Submit your manuscripts at

http://www.hindawi.com
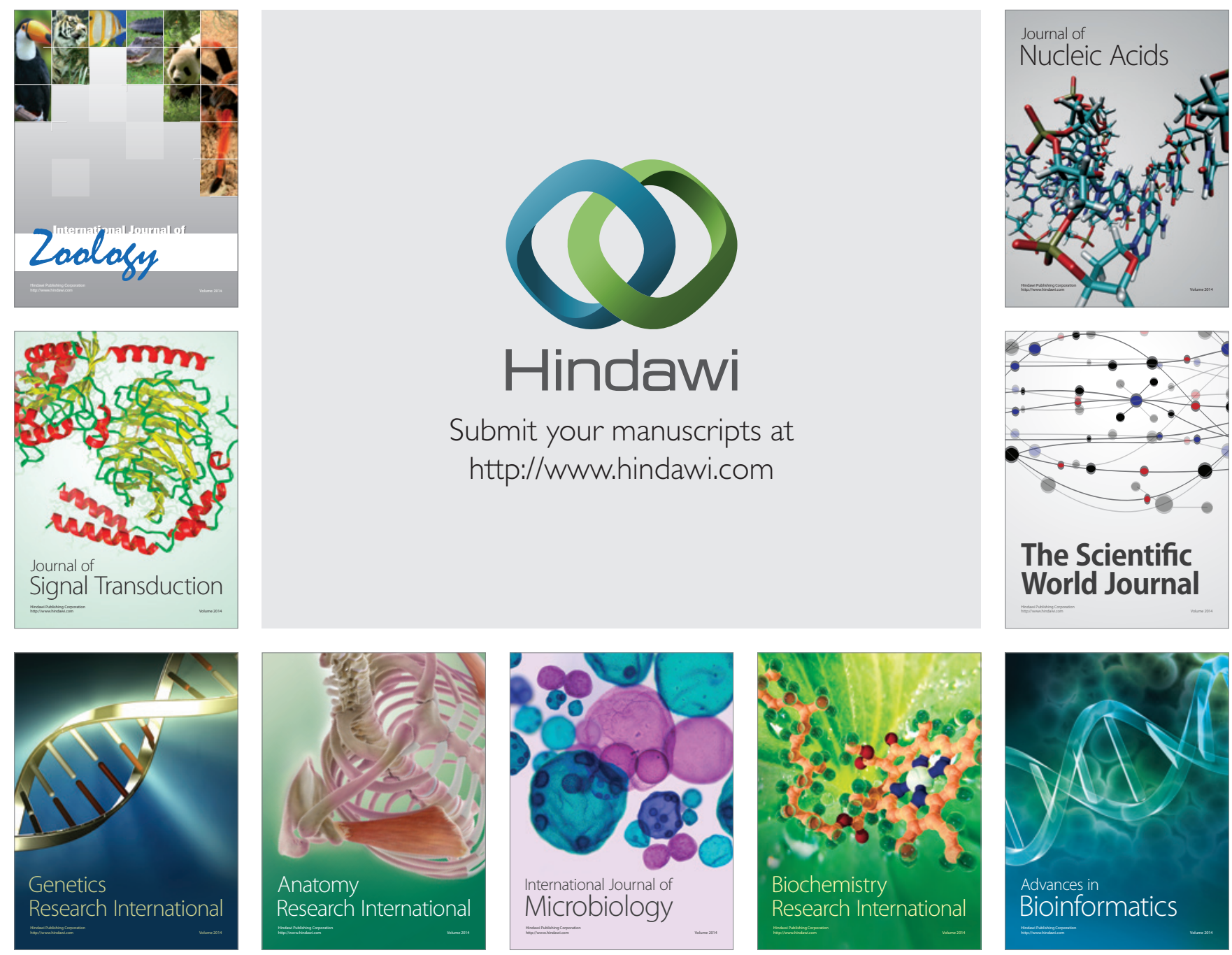

The Scientific World Journal
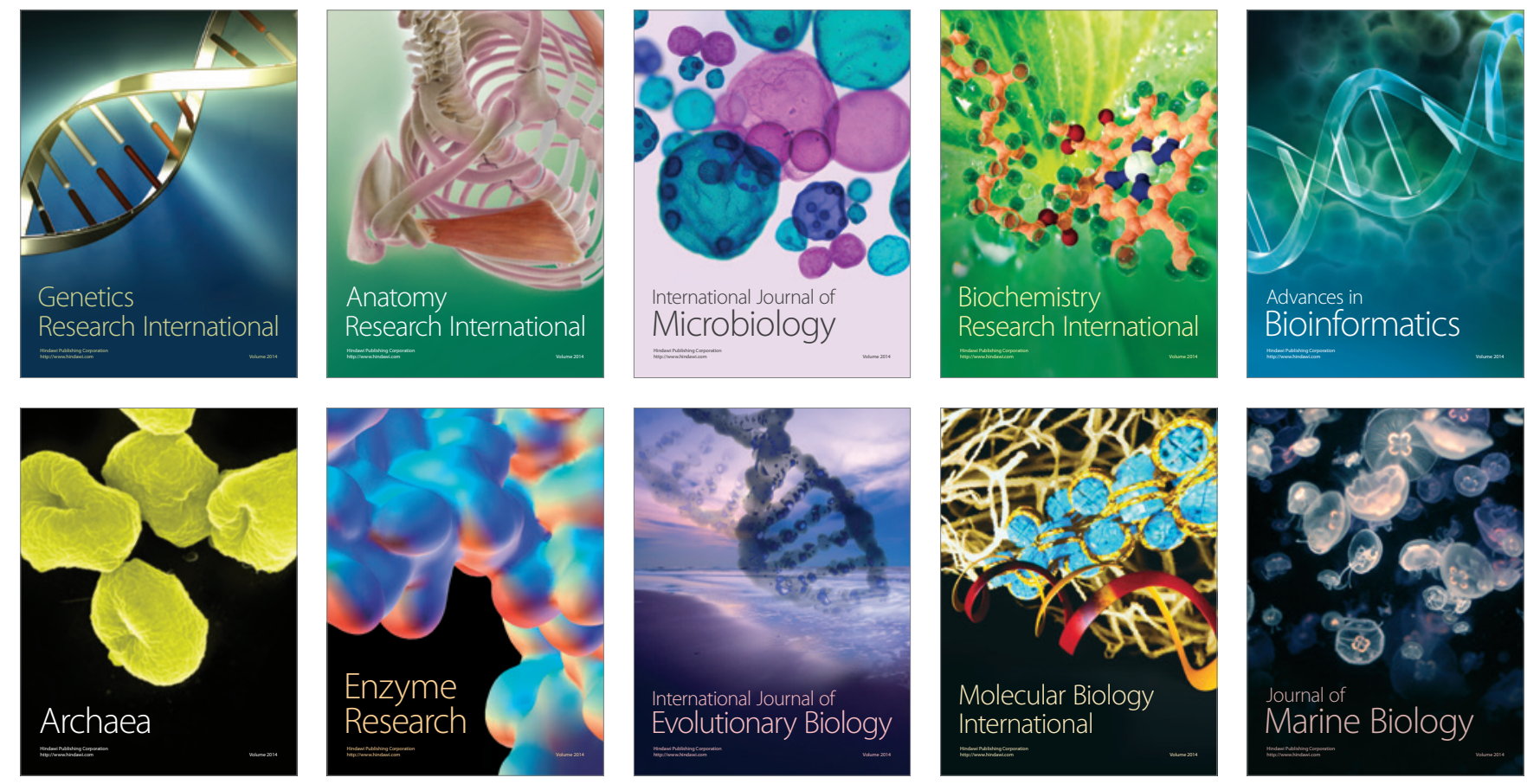